\title{
An in vitro investigation of the dose-dependent effects of glucose and fructose on intestinal de novo lipogenesis
}

\author{
S. Steenson ${ }^{1,2}$, B.A. Fielding ${ }^{1}$, A.M. Umpleby ${ }^{1}$, J.A. Lovegrove ${ }^{2}$ and K.G. Jackson ${ }^{2}$ \\ ${ }^{1}$ Department of Nutritional Sciences, Faculty of Health and Medical Sciences, University of Surrey, Guildford, GU2 \\ $7 W G, U K$ and \\ ${ }^{2}$ Department of Food \& Nutritional Sciences and Institute for Cardiovascular and Metabolic Research (ICMR), \\ University of Reading, Reading RG6 6AP, UK
}

The consumption of the monosaccharide fructose has been linked to several deleterious health effects, including post-prandial hypertriglyceridaemia ${ }^{(1)}$, which is an independent risk factor for cardiovascular disease ${ }^{(2)}$. A lack of regulation in the metabolism of fructose, as compared to glucose, may favour its use as a substrate for the synthesis of new fatty acids via de novo lipogenesis (DNL), thus contributing to both intracellular and circulating lipid pools. While much research has focussed on the effects of fructose in stimulating hepatic DNL, there is also evidence that intestinal enterocytes are capable of DNL, utilising fructose as the substrate ${ }^{(3)}$. The current study compared the production of de novo palmitate from varying concentrations, as well as a mixture, of glucose and fructose within an in vitro model of the small intestine.

Caco-2 cells (passage 49-53) were grown for 21 days on Transwell ${ }^{\circledR}$ permeable supports, creating separate apical (lumenal) and basolateral (circulation) compartments. Cells were treated for $96 \mathrm{~h}$ with $5 \mathrm{mM}, 25 \mathrm{mM}, 50 \mathrm{mM}$, or a $25 \mathrm{mM}$ mixture $(12.5 \mathrm{mM}$ of each) of glucose and fructose, in addition to a $0.5 \mathrm{mM}$ fatty acid-taurocholate micelle mixture, representing the habitual dietary fat composition of the UK population ${ }^{(4)}$. Treatment media contained $20 \%(\mathrm{v} / \mathrm{v}){ }^{13} \mathrm{C}_{6}$-glucose or ${ }^{13} \mathrm{C}_{6}$-fructose isotopes to trace the conversion of each sugar into triglyceride (TG) palmitate, which was quantified in both intracellular and secreted (basolateral) $\mathrm{TG}$ fractions via gas chromatography mass spectrometry.

This is the first time that DNL has been demonstrated within Caco-2 cells, a commonly used in vitro model of the human intestine, with both fructose and glucose utilised as substrates for DNL. Increasing the fructose (intracellular and secreted) and glucose (intracellular only) concentration lead to significantly higher amounts of de novo palmitate synthesis (Fig. 1). Within a mixture of sugars, glucose appears to be the preferred DNL substrate. Further work is needed to determine the possible mechanisms underlying these differences, including alterations in the gene expression and funtioning of key proteins involved in TG metabolism, such as microsomal triglyceride transfer protein. These data provide novel in vitro evidence of DNL within human intestinal cells, thus supporting current UK and US government recommendations to limit the intake of dietary calories from free sugars.

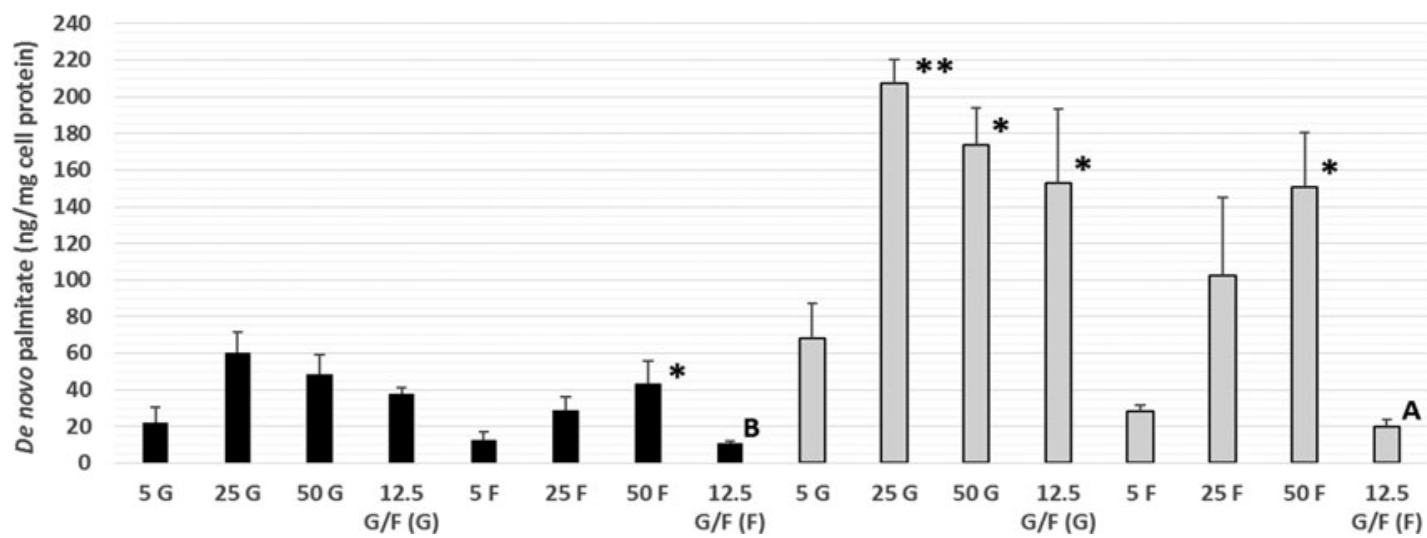

Fig. 1. Amount of de novo palmitate (ng/mg cell protein) synthesised from glucose $(\mathrm{G})$ or fructose (F) in secreted (black bars) and intracellular (grey bars) TG. Values are means $(n=3$ independent experiments) \pm SEM (tracer type in parentheses). Secreted and intracellular TG-palmitate for each of the glucose and fructose treatments were analysed separately by one-way ANOVA (LSD post-hoc) and independent $\mathrm{t}$-test: $* \mathrm{P}<0.05, * * \mathrm{P}<0.01 \mathrm{vs}$. $5 \mathrm{G}$ or 5 F. A $(\mathrm{P}<0.05), \mathbf{B}(\mathrm{P}<0.005)$ vs. $12.5 \mathrm{G} / \mathrm{F}(\mathrm{G})$.

1. Stanhope KL, Bremer AA, Medici V et al. (2011) J Clin Endocrinol Metab 96, E1596-1605.

2. Eberly LE, Stamler J, Neaton JD (2003) Arch Intern Med 163, 1077-1083.

3. Haidari M, Leung N, Mahbub F et al. (2002) J Biol Chem 277, 31646-31655.

4. Pot GK (2012) Br J Nutr 107, 405-415. 